\title{
An Investigation on the Nature of Wireless Scheduling
}

\author{
Cem Boyac1, Bo Li, and Ye Xia \\ Computer and Information Science and Engineering Department \\ University of Florida
}

\begin{abstract}
The paper studies the complexity of the wireless scheduling problem under interference constraints. We first relate the definition of the capacity region to the weighted fractional coloring problem. Then, the scheduling-for-stability problem under deterministic arrivals is studied in light of this relationship. We emphasize the requirement that the scheduling algorithm uses a tractable amount of processing and storage resources. Two classes of algorithms are defined and a complexity result is derived for the intersection of the two classes. We also exhibit an algorithm that can achieve the storage requirement by relaxing the processing requirement. The results are used to examine interesting sections of the capacity region. Finally, we relate the new interpretation and theory about the capacity region to the notion of set $\sigma$-local pooling.
\end{abstract}

\section{INTRODUCTION}

Recent development in wireless communication coupled with advancing memory capacity and processing speed has opened a new era of research that strives to enhance data transfer in multiple dimensions. Although the last decade witnessed fast improvement in the capability and cost effectiveness of radio transceivers, efficiency of wireless communication systems still heavily depends on the methodology to improve the utilization of the shared wireless medium.

Wireless interference limits simultaneous access to the medium by multiple devices. Various interference models have been developed to capture that limitation. One of the most popular models is known as the protocol model [1]. In this model, interference between links is captured by a symmetric 0-1 matrix. For a link set $E$, the $(i, j)^{t h}$ entry of the $|E| \times|E|$ interference matrix is 1 if links $i$ and $j$ cannot transmit simultaneously and both succeed, and 0 otherwise. Equivalently, the interference relationship can also be represented by an interference graph (or conflict graph), in which a node represents a physical link and an edge represents the existence of interference between two physical links. This paper primarily focuses on the protocol model. However, it is likely to also have relevance to other popular interference models, such as the physical model [2].

Given an interference model, an important area of research, to which this paper contributes, investigates how to schedule the transmissions of the links so that some chosen objective can be achieved. For simplicity, the paper considers a timeslotted system where time is divided into fixed-sized slots, the packets are all identical in size, and each link has the capacity of transmitting one packet in a time slot if access is granted. Under the protocol model, a feasible schedule is a subset of the links such that no two links in the set interfere with each other. Such a set of links can be activated for simultaneous transmission on the same time slot. Note that a schedule is feasible if and only if it corresponds to an independent set of the interference graph.

A canonical scheduling problem is as follows. Suppose the arrival rates of traffic to the links fall into the capacity region [3], which, loosely speaking, is the set of arrival rates that are "schedulable" by some algorithm. Find a scheduling policy, which can produce a feasible schedule in each time slot, so that the network queues remain stable. Various definitions of stability have been (or may be) developed, such as the queues being bounded or the Markovian queue processes being positive recurrent.

The motivation of this study is to understand the inherent complexity of the scheduling-for-stability problem. Many authors have studied various versions of this scheduling problem. The problem appears to be very difficult in general. Tassiulas and Ephremides showed that if a schedule corresponding to a maximum weight independent set (MWIS) is used in each time slot, then the network of queues can be stabilized for any arrival rate vector inside the capacity region (in which case, we say the capacity region is achieved), for a class of Markov processes [4]. Finding an MWIS is a very difficult problem in itself (e.g., strongly NP-hard and cannot be approximated by a constant ratio). This has led to the belief that the overall scheduling problem is difficult. However, we have found very few studies that address the inherent difficulty of the overall scheduling problem (as opposed to the one-step sub-problem on each time slot). Later, Tassiulas showed that the capacity region can be achieved by a simpler probabilistic algorithm running in each time slot [5]. However, he did comment informally that the probabilistic algorithm tries to solve the MWIS sub-problem but over multiple time slots. The comment was later formalized and generalized in [6]. At this point, one might hypothesize that the independent set sub-problem is unavoidable and any scheduling policy that employs a simple one-step (per-time-slot) algorithm must pay for the inherent cost of scheduling in other ways. Most likely, the cost is merely amortized over time, and this may lead to poor transient performance of the policy, such as large queues or slowness in reaching system steady state.

Recent development has made it even more relevant to study the inherent complexity of scheduling. Much of the latest work concentrates on devising even simpler and decentralized one- 
step algorithms that achieve the full capacity region [7] [8] [9] [10] [11] [12] [13]. However, if the complexity of the scheduling problem is irreducible, then one may wonder where the hidden cost is in these algorithms. A related stream of wireless scheduling research concentrates on devising simple one-step algorithms that are guaranteed to achieve some subsets of the capacity region, for an arbitrary network [14] [15] [16] [17] [18] [19]. Again, a complexity question exists here: Is the scheduling problem inherently easy on each of these subsets? In [20] [21] [22], the authors showed that, if the interference graph satisfies a condition called local pooling, then the Longest Queue First (LQF) policy (which is simple) achieves the full capacity region. But, it is unknown whether the scheduling problem for this special class of networks is easy or not. In [18], the authors defined the $\sigma$-local pooling factor for an arbitrary network, where $0<\sigma \leq 1$, and showed that LQF achieves $\sigma \Lambda$, where $\Lambda$ is the capacity region.

Several studies have implicitly addressed similar concerns of this paper. In particular, the authors of [6] examined the tradeoffs among the complexity of the one-step algorithm, the achievable rate region and the delay. There, the onestep algorithm was assumed to be the MWIS schedule or its approximations. The delay and complexity relationship was also briefly surveyed. Other papers that examined the delay issue of scheduling include [23], [24], [25]. They in fact examined the queue sizes instead of the actual delays. The upper bound of the queue sizes determines the delay property. Some linear trade-offs were observed between delay and complexity.

The following is a summary of our main results and contributions. We start with formulating the capacity region using the fractional coloring problem. Although in [26] a similar study is conducted, we concentrate on a harder setting where a (maximal) feasible schedule at each step is a maximal independent set of the interference graph. We next consider the scheduling-for-stability problem under deterministic arrivals and study its complexity. We incorporate the queue size bound into the complexity results. We show that, in general, it is impossible to have small queue sizes using simple one-step algorithms unless ZPP = NP. To the best of our knowledge, this is one of the few formal connections to the theory of computational complexity for the stability problems. We also use the approach in [4] to demonstrate that an algorithm that uses an MWIS solver as a one-step subroutine is storage-wise efficient. We then examine the geometry of the capacity region. Finally, we relate the fractional coloring problem and the new interpretation of the capacity region to the notion of set $\sigma$-local pooling. We believe that the results of this paper are useful for continued investigation on the inherent complexity of wireless scheduling.

This paper is structured as follows. In Section II, we provide the notation and definitions of related problems. In Section III, we define the capacity region in terms of the fractional coloring problem, and hence, connect scheduling to coloring. In Section IV, we analyze the complexity of efficiently stabilizing a network under deterministic arrivals.
We show that the MWIS-based algorithm is storage-wise efficient in Section V. In Section VI, we investigate different interesting sections of the capacity region. In Section VII, we relate fractional coloring and $\sigma$-local pooling. We conclude our work and discuss future research directions in Section VIII.

\section{Preliminaries}

We consider a wireless network with one-hop traffic. That is, any data is transmitted only once, and after that, it leaves the network. We model the wireless link set $L$ 's interference relations with an interference graph, $G=(V, E)$, where each link $l \in L$ in the original network corresponds to a node $v \in V$. Two nodes $v_{1}, v_{2} \in V$ are connected in $G$, if and only if the corresponding links in the original network interfere with each other. We assume a symmetric interference relation thus $G$ is undirected. A feasible schedule is defined to be a set of non-interfering nodes in $G$. A maximal schedule is a feasible schedule that cannot include any more nodes without violating the feasibility constraint. A feasible schedule in a network corresponds to an independent set in $G$. The set of all feasible schedules is denoted by $I_{L}$; this set can also be interpreted as all independent sets of the graph $G$. We denote the set of all maximal schedules by $M_{L}$, and use $m \in M_{L}$ to denote a single maximal schedule. Throughout the paper, we use the word schedule to refer to a feasible maximal schedule. When applicable, $M_{L}$ is regarded as a $|V| \times\left|M_{L}\right|$ 0-1 matrix. Each column of the matrix is a $0-1$ vector representation of a maximal independent set of $G$, with 1 indicating that the corresponding node (in $G$ ) is selected (and the link is active in the schedule) and 0 otherwise. A column vector that has 1 's in all entries is denoted by $\vec{e}$.

We adopt the conventional definition of the capacity region:

$$
\Lambda=\left\{\lambda \mid 0 \leq \lambda \leq \mu \text { for some } \mu \in \operatorname{Co}\left(M_{L}\right)\right\} .
$$

The interior of the capacity region is defined as:

$$
\Lambda^{o}=\left\{\lambda \mid 0 \leq \lambda<\mu \text { for some } \mu \in \operatorname{Co}\left(M_{L}\right)\right\} .
$$

Note that we can state: For any rational arrival rate vector $\lambda=\left(\lambda_{1}, \lambda_{2}, \ldots, \lambda_{|V|}\right)^{\prime}$ in the capacity region, there exists a coefficient vector $\alpha \in \mathbb{Q}^{|V|}$ such that $M_{L} \alpha \geq \lambda$, where $\alpha \geq 0$ and $\sum_{i=1}^{\left|M_{L}\right|} \alpha_{i} \leq 1$. $^{1}$

\section{A. Wireless Link Scheduling}

The wireless scheduling problem is typically about stabilizing the queues of the links under some arrival traffic pattern. Depending on the precise definition, stability may mean the network queues being bounded or the Markovian queue processes being positive recurrent [6]. Various definitions of stability are often related to each other under technical conditions. In this paper, we step back a little and define a more basic version of the scheduling problem. It is our belief that this basic version contains essential elements about the complexity of various stability problems.

\footnotetext{
${ }^{1}$ We use the convention that, for a vector $x, x_{i}$ denotes its $i$-th component.
} 
We now define a static version of the scheduling problem. Given an arrival rate vector, the scheduling problem is to find a convex combination of the schedules such that the resulting service rate dominates the arrival rate. Formally, given a (rational) arrival rate vector $\lambda$, find a non-negative timeshare vector $\alpha$ such that $M_{L} \alpha \geq \lambda$ and $\sum_{i=1}^{\left|M_{L}\right|} \alpha_{i} \leq 1$ (faster than unit time).

Our conjecture is that the complexity of various schedulingfor-stability problems is determined by the shape of the convex polytope of the above static feasibility problem. For instance, if $\lambda$ is strictly inside the capacity region and if we can find a (service rate) vector that strictly dominates $\lambda$ and satisfies the above feasibility problem, then the arrival traffic can be stabilized for nearly all definitions of stability encountered in the literature. Hence, the focus of the paper is about this static problem and its variants.

We use $\alpha^{\lambda}$ to denote a solution to the above scheduling problem. The problem and solutions both depend on the graph $G$. Here, we suppress the dependence on $G$ in the notation.

\section{B. Fractional Coloring Problem}

The chromatic number of a graph $G, \chi(G)$, is the minimum number of colors to paint the vertices so that the connected nodes do not share the same color. Due to its relation to the vertex covering problems, the chromatic number problem has been extensively studied in the graph theory field. The chromatic number problem is NP-complete. It can be represented with an integer programming (IP) formulation. A linear programming (LP) relaxation of this IP, which is known as the fractional coloring problem, is also central to the fractional packing and covering problems in graphs. Here, we provide a formulation for the weighted fractional coloring problem. Let $w \in \mathbb{Q}^{|V|}$ be a (component-wise) non-negative weight vector.

$$
\begin{aligned}
\chi_{f}(G, w)=\operatorname{minimize} & \sum_{m \in M_{L}} \alpha_{m} \\
\text { subject to } & M_{L} \alpha \geq w \\
& \alpha \geq 0 .
\end{aligned}
$$

The optimal value $\chi_{f}(G, w)$ is called the weighted fractional chromatic number. We let $\beta^{w} \in \mathbb{Q}^{|V|}$ denote a solution to the fractional coloring problem.

The weighted fractional chromatic number of a given interference graph in the wireless scheduling context can be interpreted as the fastest way of serving queued data (without additional arrivals) in the amount given by $w$ if we can hop between schedules in infinitesimal time slots. In [26], a similar problem is studied for the 1-hop interference model, where the schedules are maximal matchings. The authors showed that, under that setting, the scheduling problem admits polynomial algorithms. In [26], three interpretations of the optimization problem are discussed. Although this work concentrates on the independent set polytope, the first interpretation in [26] and our perspective are closely related.

\section{Minimum Span Packet Scheduling}

Given an interference graph $G$ and initial queue sizes at all the links, denoted by the vector $w$, we define the minimum span packet scheduling (MSPS) problem to be the smallest number of time slots to drain the packets from the queues under the interference constraints and unit transmission capacity at each link. The following is an IP formulation of this problem:

$$
\begin{aligned}
\Gamma(G, w)=\operatorname{minimize} & \sum_{m \in M_{L}} c_{m} \\
\text { subject to } & M_{L} c \geq w \\
& c_{m} \in \mathbb{Z}^{+}
\end{aligned}
$$

where $\mathbb{Z}^{+}$denotes non-negative integers.

Now, consider refining the time slots while keeping the initial amount of queued data fixed. When reducing the size of the time slots, we also make the unit packet length smaller. If we choose the time slot to be $R$ times smaller, then the initial queue sizes will become $R$ times larger in terms of packet count. The optimization problem can be written as follows.

$$
\begin{aligned}
& \Gamma(G, w, R)=\operatorname{minimize} \sum_{m \in M_{L}} c_{m} \\
& \text { subject to } M_{L} c \geq R w \\
& c_{m} \in \mathbb{Z}^{+} .
\end{aligned}
$$

The following lemma indicates if we make the time slot size infinitesimally small and scale the optimal value down by the same scaling factor, the solution of the above problem will converge to the solution of the fractional coloring problem.

Lemma 1. $\lim _{R \rightarrow \infty} \frac{\Gamma(G, w, R)}{R}=\chi_{f}(G, w)$.

Proof: Let $\beta^{w}$ be a solution corresponding to $\chi_{f}(G, w)$. Since $w \in \mathbb{Q}^{|V|}$ and is non-negative, we can conclude $\beta^{w} \in$ $\mathbb{Q}^{\left|M_{L}\right|}$ and is non-negative. We denote the reduced form of each rational number $\beta_{i}^{w}$ by $\frac{a_{i}}{b_{i}}$ for some $a_{i}, b_{i} \in \mathbb{Z}^{+}, b_{i} \neq 0$, where $a_{i}$ and $b_{i}$ are relatively prime. Let the least common multiple of the $b_{i}$ 's be $B$. Similarly, denote the reduced form of each $w_{i}$ by $\frac{c_{i}}{d_{i}}$ for some $c_{i}, d_{i} \in \mathbb{Z}^{+}, d_{i} \neq 0$, where $c_{i}$ and $d_{i}$ are relatively prime. Denote the least common multiple of the $d_{i}$ 's by $D$. For any $j \in \mathbb{Z}^{+}$and $j \geq 1$, the optimization problem for deriving $\chi_{f}(G, j B D w)$ has an integer optimal solution, and hence, $\chi_{f}(G, j B D w)=\Gamma(G, w, j B D)$. Now suppose $k B D \leq R<(k+1) B D$ for some $k \in \mathbb{Z}^{+}$. Note that

$$
\Gamma(G, w, k B D) \leq \Gamma(G, w, R) \leq \Gamma(G, w,(k+1) B D) .
$$

Hence,

$$
\chi_{f}(G, k B D w) \leq \Gamma(G, w, R) \leq \chi_{f}(G,(k+1) B D w) .
$$

Dividing each term above by $R$ and using the linearity of $\chi_{f}(G, w)$ in $w$, we have

$$
\chi_{f}\left(G, \frac{k B D w}{R}\right) \leq \frac{\Gamma(G, w, R)}{R} \leq \chi_{f}\left(G, \frac{(k+1) B D w}{R}\right) .
$$


Using the continuity of $\chi_{f}(G, w)$ in $w$, as $R \rightarrow \infty$, both $\chi_{f}\left(G, \frac{k B D w}{R}\right)$ and $\chi_{f}\left(G, \frac{(k+1) B D w}{R}\right)$ approach $\chi_{f}(G, w)$.

A similar result for the non-weighted case was derived in [27] where the authors studied how to minimize the spectrum usage in FDMA networks.

\section{Relevance of Fractional Coloring to SCHEDULING}

Under the protocol model, the scheduling problem assumes a strong combinatorial structure. In this section, we will bridge scheduling with the fractional coloring problem to highlight the combinatorial nature. The results will be useful for the following sections. The next theorem states that the capacity region can be defined in terms of the weighted fractional chromatic number, where the weight vector $\lambda$ is the arrival rates on the links.

\section{Theorem 1.}

$$
\Lambda=\left\{\lambda \mid 0 \leq \chi_{f}(G, \lambda) \leq 1\right\} .
$$

Proof: Take any arrival vector $\lambda \in \Lambda$. By definition, there exists $\mu \in C o\left(M_{L}\right)$ such that $\lambda \leq \mu$. Since $\mu \in C o\left(M_{L}\right)$, there exists $\beta$ such that $M_{L} \beta=\mu$, where $\beta \geq 0$ and $\sum_{i=1}^{\left|M_{L}\right|} \beta_{i}=1$. Hence, $\beta$ is a feasible solution to the optimization problem for $\chi_{f}(G, \lambda)$ and its components sum to 1 . Therefore, $\chi_{f}(G, \lambda) \leq 1$. For the reverse direction, take an arrival vector $\lambda$ such that $0 \leq \chi_{f}(G, \lambda) \leq 1$. Assume $\beta$ is a solution corresponding to $\chi_{f}(G, \lambda)$. That is, $M_{L} \beta \geq \lambda$ and $B=\sum_{i=1}^{\left|M_{L}\right|} \beta_{i} \leq 1$. If $B=0$, then $\lambda=0$, which is in $\Lambda$. If $B \neq 0$, then $M_{L} \frac{\beta}{B} \geq \lambda$ and $M_{L} \frac{\beta}{B} \in C o\left(M_{L}\right)$. This indicates $\lambda \in \Lambda$.

By the proof for Theorem 1, we conclude that a solution to the fractional coloring problem can be thought as a timesharing vector of schedules for the scheduling problem.

Similarly, we can define the boundary of the capacity region in terms of the weighted fractional chromatic number. But, we need a technical fact first.

Fact 1 For any $J \subseteq L$, there exists $\mu \in C o\left(M_{L}\right)$ such that $\mu_{j}>0$ for all $j \in J$.

The proof is simple and is omitted. Next,

Theorem 2. $\lambda \in\left(\Lambda-\Lambda^{o}\right) \Longleftrightarrow \chi_{f}(G, \lambda)=1$.

Proof: We first show if $\lambda \in\left(\Lambda-\Lambda^{\circ}\right)$, then $\chi_{f}(G, \lambda)=1$. Since by Theorem $1, \chi_{f}(G, \lambda) \leq 1$ for all $\lambda \in \Lambda$, we only need to show when $0 \leq \chi_{f}(G, \lambda)<1, \lambda \in \Lambda^{o}$.

If $\chi_{f}(G, \lambda)=0$, then $\lambda=0$ and hence $\lambda \in \Lambda^{o}$. Consider the case $0<\chi_{f}(G, \lambda)<1$. Let the corresponding optimal solution be $\beta$ and $B=\chi_{f}(G, \lambda)=\sum_{i=1}^{\left|M_{L}\right|} \beta_{i}$. Denote $\mu=$ $M_{L} \frac{\beta}{B}$ and $\nu=M_{L} \beta$. Note that $\mu \in C o\left(M_{L}\right)$ and $\mu \geq \nu \geq \lambda$. Let $J \subseteq L$ be largest set such that $\nu_{j}=0$ for all $j \in J$. Then, $\mu_{j}=\nu_{j}=\lambda_{j}=0$ for $j \in J ; \mu_{j}>\nu_{j} \geq \lambda_{j}$ for $j \notin J$. Now, take any $\hat{\mu} \in \operatorname{Co}\left(M_{L}\right)$ with the property $\hat{\mu}_{j}>0$ for $j \notin J$. This is possible by Fact 1 . Construct a new vector in $\operatorname{Co}\left(M_{L}\right)$ : $\tilde{\mu}=(1-\epsilon) \mu+\epsilon \hat{\mu}$, where $0<\epsilon<1$. When $\epsilon$ is sufficiently small, we can have $\tilde{\mu}>0$ and $\tilde{\mu}>\lambda$. Hence, $\lambda \in \Lambda^{\circ}$.
Next, suppose $\chi_{f}(G, \lambda)=1$. By Theorem $1, \lambda \in \Lambda$. Suppose $\lambda$ is in $\Lambda^{o}$. Then, there exists $\mu \in \operatorname{Co}\left(M_{L}\right)$ such that $\mu>\lambda$. This in turn implies that there exists $\epsilon>0$ such that $\mu>(1+\epsilon) \lambda$, or $\frac{1}{1+\epsilon} \mu>\lambda$. Suppose $\alpha$ satisfies $M_{L} \alpha=\mu$, where $\alpha \geq 0$ and $\sum_{i=1}^{\left|M_{L}\right|} \alpha_{i}=1$. Then, $\frac{1}{1+\epsilon} \alpha$ is a feasible solution to the optimization problem for finding $\chi_{f}(G, \lambda)$, but its components sum to less than one. This contradicts the assumption $\chi_{f}(G, \lambda)=1$. Therefore $\lambda \notin \Lambda^{o}$.

Corollary 1. $\Lambda^{o}=\left\{\lambda \mid 0 \leq \chi_{f}(G, \lambda)<1\right\}$.

An interpretation of the theorems and corollary above is as follows. The capacity region consists of vectors that can be serviced in unit time or less by some mixing of schedules. The interior contains vectors that are schedulable in strictly less than unit time.

The boundary of the capacity region, $\Lambda-\Lambda^{\circ}$, consists of vectors that leave the capacity polytope when multiplied by a scalar of the form $1+\epsilon$ for any $\epsilon>0$. The following formalizes this interpretation of the boundary.

Lemma 2. $\lambda \in\left(\Lambda-\Lambda^{o}\right) \Longleftrightarrow \lambda \in \Lambda$ and $\lambda(1+\epsilon) \notin \Lambda$ for all $\epsilon>0$.

Proof: By Theorem 2, $\lambda \in\left(\Lambda-\Lambda^{o}\right)$ implies $\chi_{f}(G, \lambda)=$ 1. Suppose $\lambda(1+\epsilon) \in \Lambda$ for some $\epsilon>0$. By the linearity of $\chi_{f}(G, w)$ in $w, \chi_{f}(G, \lambda(1+\epsilon))=(1+\epsilon) \chi_{f}(G, \lambda)$. By Theorem $1,0 \leq(1+\epsilon) \chi_{f}(G, \lambda) \leq 1$, which implies $\chi_{f}(G, \lambda) \leq \frac{1}{1+\epsilon}$, contradicting the assumption. Conversely, suppose $\lambda \in \Lambda$ and there is no $\epsilon>0$ such that $\lambda(1+\epsilon) \in \Lambda$. Suppose $\lambda \in \Lambda^{o}$. Then, there exists $\mu \in \operatorname{Co}\left(M_{L}\right)$ such that $\mu>\lambda$. Hence, there exists $\epsilon>0$ such that $\mu>(1+\epsilon) \lambda$. Then, $(1+\epsilon) \lambda \in \Lambda$, which is a contradiction.

A vector inside the capacity region becomes increasingly difficult to service as it approaches the boundary. Typical suboptimal scheduling algorithms in the literature achieve a part of the capacity region derived by scaling the capacity region uniformly by some less-than-1 constant (e.g., [14], [17], [18]). Motivated by this fact, we associate a number with every arrival rate vector.

Definition 1. For any non-negative rate vector $\lambda$, let

$$
\psi_{\lambda}^{*} \triangleq \sup \{\psi \mid \psi \lambda \in \Lambda\} .
$$

Note that $\psi_{\lambda}^{*}=1$ if and only if $\lambda$ is on the boundary of the capacity region. If $\lambda \in \Lambda$, a larger value of $\psi_{\lambda}^{*}$ indicates $\lambda$ is deeper inside the capacity region. We next demonstrate the relationship between this number and the weighted fractional chromatic number.

Lemma 3. For a non-negative vector $\lambda, \chi_{f}(G, \lambda)=\frac{1}{\psi_{\lambda}^{*}}$.

Proof: By linearity, we have $\chi_{f}\left(G, \psi_{\lambda}^{*} \lambda\right)=\psi_{\lambda}^{*} \chi_{f}(G, \lambda)$. By Lemma $2, \psi_{\lambda}^{*} \lambda$ is on the boundary of the capacity region. By Theorem 2, $\chi_{f}\left(G, \psi_{\lambda}^{*} \lambda\right)=1$. Hence, $\psi_{\lambda}^{*} \chi_{f}(G, \lambda)=1$.

This result associates the fractional coloring problem with the difficulty of servicing an arrival rate vector. Note that a solution to the fractional coloring problem is also a solution to the scheduling problem (which is a feasibility problem) 
but not vice versa. Accordingly, we turn to the question of how scheduling (and hopefully, achieving stability) and the weighted fractional coloring problem are related when an arrival rate vector is close the boundary. The following lemma indicates that a solution to the scheduling problem approaches a solution to the fractional coloring problem as the rate vector gets sufficiently close to the boundary.

Lemma 4. For an arrival vector $\lambda \in \Lambda$, any $\alpha^{\lambda}$ (see the definition of this notation in Section II-A) is a $\psi_{\lambda}^{*}$ approximation to the optimization problem for $\chi_{f}(G, \lambda)$.

Proof: By Lemma 3, $\chi_{f}(G, \lambda)=\frac{1}{\psi_{\lambda}^{*}}$. Now take any solution of the scheduling problem, $\alpha^{\lambda}$, by definition $\alpha^{\lambda}$ is a feasible solution to the problem for finding $\chi_{f}(G, \lambda)$ and $\sum_{i=1}^{\left|M_{L}\right|} \alpha_{i}^{\lambda} \leq 1=\chi_{f}(G, \lambda) \psi_{\lambda}^{*}$. This concludes the proof.

One implication is that an algorithm that stabilizes an arrival rate vector yields an approximation of the weighted fractional coloring problem ${ }^{2}$. Furthermore, the approximation ratio tends to 1 as the arrival rate vector gets closer to the boundary. The result establishes a partial equivalence between the two problems. They are the same for the vectors near or on the boundary, but are potentially very different for vectors further from the boundary.

The (unweighted) fractional coloring number of a graph $G$, denoted by $\chi_{f}(G)$, is the solution to the weighted version when the weight vector consists of all ones, i.e., $\chi_{f}(G)=$ $\chi_{f}(G, \vec{e})$. Consider the problem of achieving the maximum identical rate on all links. We denote this rate by $\zeta(G)$. The following is the LP formulation for finding $\zeta(G)$.

$$
\begin{gathered}
\zeta(G)=\operatorname{maximize} \\
\text { subject to } M_{L} \alpha \geq z \vec{e} \\
z, \alpha \geq 0 .
\end{gathered}
$$

Lemma 5. $\zeta(G)=\frac{1}{\chi_{f}(G)}$.

Proof: By Lemma 2 and the optimality, $\zeta(G) \vec{e}$ is on the boundary of $\Lambda$. By Theorem 2, we conclude $\chi_{f}(G, \zeta(G) \vec{e})=$ 1. Observe $\chi_{f}(G, \zeta(G) \vec{e})=\zeta(G) \chi_{f}(G, \vec{e})=1$.

\section{Complexity of Scheduling Problem}

In Section III, we established a connection between the fractional coloring and the scheduling problems. Next, we will use the derived results to reveal complexity characteristics of the scheduling problem and stable algorithms. We will focus on a deterministic system, where the arrivals per time slot are constant for every link. The arrival rates are assumed to be in $\mathbb{Q}^{|L|}$ and all links have unit transmission capacity.

A stable algorithm in this setting is defined to be any algorithm that, given initially empty queues, keeps the queue sizes bounded throughout the execution for all arrival rates in the interior of the capacity region. For a stable algorithm, the upper bound on the queue sizes might be a function of the arrival rate and the encoding length of the problem. Later, we

\footnotetext{
${ }^{2}$ Strictly speaking, we only showed the solution to the static scheduling problem gives such an approximation.
}

will relate the upper bound to the distance to the boundary for each arrival rate.

The existence of a stable algorithm implies the existence of a vector that solves the wireless scheduling problem. However, given a solution $\alpha^{\lambda}$ to the scheduling problem, applying it as an algorithm at each time slot might not be straightforward. In addition to the frequencies of the schedules, when to activate each schedule also plays a key role. Additionally, although the two are related, the notion of stability requires the condition that the system runs forever. To deal with these issues and to continue our investigation of computational complexity, we will investigate stable algorithms that not only bound the queue sizes but also ensure that the bound is polynomial in the input's encoding length.

Before we start, we define a quantity $\epsilon^{\lambda}$ for each arrival rate vector $\lambda$, which is a measure of distance to the boundary. It will be used to characterize the queue size upper bound of a stable algorithm.

$$
\epsilon^{\lambda} \triangleq \sup \{\epsilon \mid \lambda+\epsilon \vec{e} \in \Lambda\} .
$$

Definition 2. An algorithm stabilizes an arrival rate vector $\lambda$ computationally efficiently if the algorithm is stable and at each iteration, the operation of the algorithm takes a polynomial number of steps in $|L|$.

Definition 3. An algorithm stabilizes an arrival rate vector $\lambda$ $\mathscr{P}$-storage-wise efficiently if the algorithm is stable and the queue sizes are upper bounded by $\mathscr{P}\left(|L|,\left(\epsilon^{\lambda}\right)^{-1}\right)$, where $\mathscr{P}$ is a polynomial function.

Theorem 3. There is no algorithm that stabilizes all arrival rate vectors in $\Lambda^{\circ}$ computationally and $\mathscr{P}$-storage-wise efficiently for any polynomial $\mathscr{P}$, unless $Z P P=N P$.

Proof: Assume there exists an algorithm that stabilizes any deterministic arrival rate vector $\lambda \in \Lambda^{o}$ computationally and $\mathscr{P}$-storage-wise efficiently. Then, all queues are upper-bounded by $\mathscr{P}\left(|L|,\left(\epsilon^{\lambda}\right)^{-1}\right)$ during the operation of the network. Let us denote this number with $\mathscr{P}^{\lambda}$ for short. Also, each time slot takes at most $P(|L|)$ computation steps, for some polynomial function $P$. Given an approximation factor $\epsilon$, we can use the algorithm as an oracle to create a fully polynomial time approximation scheme (FPTAS) for the fractional coloring problem $\chi_{f}(G)$. (A similar technique is used for the concurrent flow problem in [28].) Assume we run the algorithm for $R$ time slots. Then, the total traffic inserted into queue $i$ is $R \lambda_{i}$ for every $i$. If all the queues are upperbounded by $\mathscr{P}^{\lambda}$, the amount of the traffic served at queue $i$ in the $R$ time slots is no less than $R \lambda_{i}-\mathscr{P} \lambda$. The average service rate at queue $i$ is at least $\frac{R \lambda_{i}-\mathscr{P}^{\lambda}}{R}$. Letting $R=\mathscr{P}^{\lambda} \epsilon^{-1}$, we ensure queue $i$ is served at an average rate $\lambda_{i}-\vec{e} \epsilon$. Therefore, we can run the stable algorithm for $\mathscr{P}^{\lambda} \epsilon^{-1}$ time slots and either achieve a timeshare vector $\alpha$ such that $M_{L} \alpha \geq \lambda-\vec{e} \epsilon$ or conclude that $\lambda$ is not in the capacity region thus creating an oracle.

By Lemma 5, the arrival rate vector $\frac{1}{\chi_{f}(G)} \vec{e}$ is on the boundary of the capacity region. By setting the precision to 
$\epsilon(6|L|)^{-1}$, we can conduct binary search and find a number $z$ such that $z \leq \frac{1}{\chi_{f}(G)} \leq z+\epsilon(2|L|)^{-1}$ using the oracle for $O\left(\log \left(\epsilon^{-1} 6|L|\right)\right)$ times. Since $1 \leq \chi_{f}(G) \leq|L|, \chi_{f}(G) \leq$ $1 / z \leq(1+\epsilon) \chi_{f}(G)$. Thus, the total running time of the algorithm is $P(|L|) O\left(\log \left(\epsilon^{-1} 6|L|\right)\right) \mathscr{P}\left(|L|, \epsilon^{-1} 6|L|\right) \epsilon^{-1} 6|L|$, which is polynomial in input $G$. However, in [29], it is proven that $\chi_{f}(G)$ cannot be approximated within $\Omega\left(|L|^{1-\epsilon}\right)$ for any $\epsilon>0$, unless ZPP $=$ NP. Therefore, devising a FPTAS here would equalize NP and ZPP as well.

The fractional coloring problem is known to be NPcomplete. Grötschel et al. [30] [31] established an equivalence between the fractional coloring and the maximum weight independent set (MWIS) problems. Formally, one can solve fractional coloring by using a solver for the MWIS problem a polynomial number of times. Additionally, an approximate solver for the MWIS problem can be used to derive an approximation algorithm for the fractional coloring problem. The approximation ratio of the MWIS solver is directly carried to the latter problem's solution [32] [33]. This result, however, should be mentioned together with the fact that the MWIS problem is NP-complete and does not admit a polynomial time approximation algorithm with an approximation ratio $|V|^{\delta}$ for some $\delta>0$ [34].

\section{Queue Dynamics Under Deterministic ArRival}

In this section, we will demonstrate a storage-wise efficient scheduling algorithm: The MWIS-based algorithm is $\mathscr{P}$ storage-wise efficient for $\mathscr{P}=\frac{3}{2}|L|\left(\epsilon^{\lambda}\right)^{-1}+\sqrt{|L|}$.

We denote the queue size of link $i \in L$ at time $t$ by $q_{i}(t)$. The arrival and departure rates at link $i$ and time $t$ are $A_{i}(t)$ and $D_{i}(t)$, respectively. Note that $A_{i}(t)=\lambda_{i}$ and $D_{i}(t)=\min \left(1, q_{i}(t)+A_{i}(t)\right) u_{i}(t)$, where $u_{i}(t)$ is an indicator function, equal to 1 if link $i$ is scheduled for transmission at time $t$ and 0 otherwise.

The system potential is defined to be the sum of all queue size squares, $\Phi(q)=\sum_{1}^{|L|} q_{i}^{2}$. We will show when the queue sizes grow beyond a threshold, the system potential drops in the next time slot. This in turn proves that the system potential is bounded and consequently the queue sizes are bounded as well.

The queue evolution is given by:

$$
q_{i}(t+1)=q_{i}(t)+A_{i}(t)-D_{i}(t) .
$$

The potential difference between times $t$ and $t+1$ is denoted by $\Delta(t) \triangleq \Phi(q(t+1))-\Phi(q(t))$. Then,

$$
\begin{aligned}
& \Delta(t) \\
= & \sum_{i \in L} q_{i}^{2}(t+1)-\sum_{i \in L} q_{i}^{2}(t) \\
= & \sum_{i \in L}\left[q_{i}(t+1)+q_{i}(t)\right]\left[q_{i}(t+1)-q_{i}(t)\right] \\
= & \sum_{i \in L}\left[2 q_{i}(t)+A_{i}(t)-D_{i}(t)\right]\left[A_{i}(t)-D_{i}(t)\right] \\
= & \sum_{i \in L}\left[2 q_{i}(t)\left(A_{i}(t)-D_{i}(t)\right)\right]+\sum_{i \in L}\left[A_{i}(t)-D_{i}(t)\right]^{2} .
\end{aligned}
$$

The second term above is bounded: $\sum_{i \in L}\left[A_{i}(t)-D_{i}(t)\right]^{2} \leq$ $|L|$. We next investigate the first term.

$$
\begin{aligned}
& \sum_{i \in L}\left[2 q_{i}(t)\left(A_{i}(t)-D_{i}(t)\right)\right] \\
= & 2 \sum_{i \in L}\left[q_{i}(t) A_{i}(t)\right]-2 \sum_{i \in L}\left[q_{i}(t) D_{i}(t)\right] .
\end{aligned}
$$

The departure vector $D(t)=\left(D_{1}(t), D_{2}(t), \ldots, D_{|L|}\right)^{\prime}$ is determined by the chosen schedule at time $t, m^{*}(t)=$ $\arg \max _{m \in M_{L}} q(t)^{\prime} m$. Note that the departure is dominated by the binary representation of the chosen schedule, $D(t) \leq$ $m^{*}(t)$.

Lemma 6. $0 \leq q(t)^{\prime} m^{*}(t)-q(t)^{\prime} D(t) \leq|L|$.

Proof: Since $q(t), m^{*}(t), D(t) \geq 0$ and $D(t) \leq m^{*}(t)$, $q(t)^{\prime} D(t) \leq q(t)^{\prime} m^{*}(t)$. As usual, we denote the $i$-th component of $m^{*}(t)$ by $m_{i}^{*}(t)$. Recall $D_{i}(t)=\min \left(1, q_{i}(t)+\right.$ $\left.A_{i}(t)\right) u_{i}(t)=\min \left(1, q_{i}(t)+A_{i}(t)\right) m_{i}^{*}(t)$. Hence,

$$
\begin{aligned}
& q_{i}(t) m_{i}^{*}(t)-q_{i}(t) D_{i}(t) \\
= & q_{i}(t) m_{i}^{*}(t)-q_{i}(t) \min \left(1, q_{i}(t)+A_{i}(t)\right) m_{i}^{*}(t) .
\end{aligned}
$$

When $q_{i}(t)=0$ or $q_{i}(t) \geq 1$ or $m_{i}^{*}(t)=0$, we have $q_{i}(t) m_{i}^{*}(t)-q_{i}(t) D_{i}(t)=0$. The only interesting case is when $0<q_{i}(t)<1$ and $m_{i}^{*}(t)=1$, where we have

$$
\begin{aligned}
& q_{i}(t) m_{i}^{*}(t)-q_{i}(t) D_{i}(t) \\
= & q_{i}(t)\left[1-\min \left(1, q_{i}(t)+A_{i}(t)\right)\right] \leq 1 .
\end{aligned}
$$

As a result,

$$
\begin{aligned}
& q(t)^{\prime} m^{*}(t)-q(t)^{\prime} D(t) \\
= & \sum_{1}^{|L|} q_{i}(t) m_{i}^{*}(t)-\sum_{1}^{|L|} q_{i}(t) \min \left(1, q_{i}(t)+A_{i}(t)\right) m_{i}^{*}(t) \\
\leq & |L| .
\end{aligned}
$$

Since we assume the arrival rate vector is in the interior of the capacity region, $A(t) \in \Lambda^{o}$, there exist $\epsilon>0$ and $\mu \epsilon$ $C o\left(M_{L}\right)$ such that $A(t)+\epsilon \vec{e} \leq \mu$. Since $q(t) \geq 0, q(t)^{\prime} A(t)+$ $q(t)^{\prime} \epsilon \vec{e} \leq q(t)^{\prime} \mu$. Moreover, $q(t)^{\prime} m^{*}(t) \geq q(t)^{\prime} \mu$ for all $\mu \in$ $\operatorname{Co}\left(M_{L}\right)$. Therefore, $q(t)^{\prime} A(t)+q(t)^{\prime} \epsilon \vec{e} \leq q(t)^{\prime} m^{*}(t)$.

$$
\begin{aligned}
& \sum_{i \in L}\left[2 q_{i}(t)\left(A_{i}(t)-D_{i}(t)\right)\right] \\
= & 2 \sum_{i \in L}\left[q_{i}(t) A_{i}(t)\right]-2 \sum_{i \in L}\left[q_{i}(t) D_{i}(t)\right] \\
\leq & 2\left[q(t)^{\prime} m^{*}(t)-q(t)^{\prime} \epsilon \vec{e}-\left(q(t)^{\prime} m^{*}(t)-|L|\right)\right] \\
\leq & -2 \epsilon q(t)^{\prime} \vec{e}+2|L| .
\end{aligned}
$$

Hence, the potential difference can be bounded as

$$
\Delta(t) \leq-2 \epsilon q(t)^{\prime} \vec{e}+3|L| .
$$

For time $t$, we denote the total queue sum as $Q(t)=$ $\sum_{i \in L} q_{i}(t)$. When $Q(t)>\frac{3|L|}{2 \epsilon}, \Delta(t)<0$. Also, if $\Phi(q(t))>$ $\left(\frac{3|L|}{2 \epsilon}\right)^{2}$, then $Q(t)>\frac{3|L|}{2 \epsilon}$. For any time $t$, the maximum 


$$
\begin{aligned}
& \vec{M}_{1}^{\prime}=(1,0,1,0,1,0) \\
& \vec{M}_{2}^{\prime}=(0,1,0,1,0,1) \\
& \vec{M}_{3}^{\prime}=(1,0,0,1,0,0) \\
& \vec{M}_{4}^{\prime}=(0,1,0,0,1,0) \\
& \vec{M}_{5}^{\prime}=(0,0,1,0,0,1)
\end{aligned}
$$

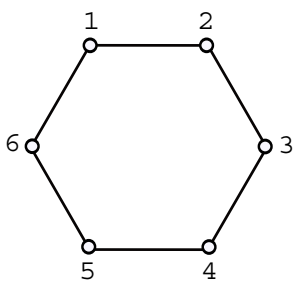

Fig. 1. Interference graph for a six-cycle network and its schedule vectors.

potential increase is bounded by $2 Q(t)+|L|$ (which is when each queue is increased by 1 ). If the potential is more than $\left(\frac{3|L|}{2 \epsilon}\right)^{2}$ at the beginning of time $t$, it will drop after one time slot. The maximum potential that the system can ever reach is bounded by $\left(\frac{3|L|}{2 \epsilon}\right)^{2}+2 \frac{3|L|}{2 \epsilon}+|L| \leq\left(\frac{3|L|}{2 \epsilon}+\sqrt{|L|}\right)^{2}$. A bounded potential translates into bounded queue sizes. The maximum queue size during the operation of the system is bounded by $\frac{3|L|}{2 \epsilon}+\sqrt{|L|}$. In conclusion, by using a solver for the MWIS problem, we can achieve a storage-wise efficient algorithm.

\section{A Closer Look to the CAPacity Region Under PROTOCOL MODEL}

In this section, we will characterize the different sections of the capacity region and provide sample arrival rate vectors that are representative of each section.

In general, the capacity region consists of arrival rate vectors that can be served by some algorithm. The boundary can be considered as the set of arrival rate vectors that are the most challenging to serve. In earlier sections, we provide two characterizations of the boundary. The first one relates to the fractional chromatic number and the second one states that the vectors on the boundary cannot be extended further and yet remain in the capacity region. By the second definition, if a vector is on the boundary, no other vector in the capacity region can dominate it in its direction. However, it is possible that, for two vectors on the boundary, one dominates the other.

Therefore, it is worth to single out the part of the boundary where a vector is not dominated by any other vector in the capacity region. This part contains the most challenging arrival rate vectors to serve. We call this region the upper-boundary of the capacity region $\Lambda$, and denote it by $\mathscr{U}(\Lambda)$. The definition of the upper-boundary excludes those vectors on the traditional boundary that are dominated by some vectors in the capacity region. Formally,

$$
\mathscr{U}(\Lambda)=\left\{\lambda \in \Lambda \mid \lambda^{\prime} \nsucceq \lambda \text { for all } \lambda^{\prime} \in \Lambda \text { and } \lambda^{\prime} \neq \lambda\right\} .
$$

The notation $¥$ means “not $\geq$ ”.

To illustrate the significance of different regions, we will use a small network and examine a few interesting arrival rate vectors. Consider the 6-cycle network whose interference graph is given in Figure 1. The schedules of this network are listed on its left.

Consider an arrival rate vector on the upper-boundary, $\left(\frac{1}{2}, \frac{1}{2}, \frac{1}{2}, \frac{1}{2}, \frac{1}{2}, \frac{1}{2}\right)^{\prime}$. This vector can be represented as a convex combination of the schedules, $\frac{1}{2} \vec{M}_{1}+\frac{1}{2} \vec{M}_{2}$. It is impossible to dominate this vector by any other convex combination of the schedules.

Next, consider the rate vector $\left(\frac{1}{2}, \frac{1}{2}, \frac{1}{2}, \frac{1}{2}, \frac{1}{2}, 0\right)^{\prime}$. It is on the boundary. However, the previous vector dominates this one. Therefore, this vector sits on the boundary but not on the upper-boundary.

Now move inside the capacity region and consider the rate vector $\left(\frac{1}{3}, \frac{1}{3}, \frac{1}{3}, \frac{1}{3}, \frac{1}{3}, \frac{1}{3}\right)^{\prime}$. We can provide two different representations of this vector: $\frac{1}{3} \vec{M}_{1}+\frac{1}{3} \vec{M}_{2}$ or $\frac{1}{2} \vec{M}_{3}+\frac{1}{2} \vec{M}_{4}+\frac{1}{2} \vec{M}_{5}$. Note that the former representation is more "efficient" than the latter since the coefficients have a smaller sum. This vector is in the convex hull of the (maximal) schedules but not on the boundary.

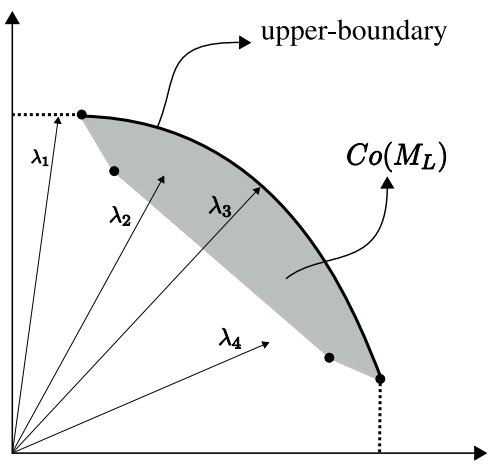

Fig. 2. Capacity region and arrival rate vectors in different sections.

In Figure 2, we provide a simplified illustration of a capacity region to demonstrate the concepts. The solid thicker line indicates the upper-boundary and the dashed line is for the rest of the boundary. $\lambda_{1}$ is on the boundary but also dominated by some vector in the capacity region. Therefore, $\lambda_{1}$ is not on the upper-boundary. $\lambda_{3}$ cannot be dominated by any other vector in the capacity region and it is on the upper-boundary. $\lambda_{2}$ is a convex combination of the schedules, but it can still be scaled up without leaving the capacity region; hence, it is both in $\operatorname{Co}\left(M_{L}\right)$ and in the interior of the capacity region. $\lambda_{4}$ is also in the interior of the capacity region but it is not in $C o\left(M_{L}\right)$. $\lambda_{4}$ has relatively low arrival rates; it may be easy to schedule such an arrival rate vector.

\section{CAPACity Region and Local Pooling}

The shape of the capacity region can affect the difficulty of the scheduling problem. Since all the other vectors in the capacity region are dominated by the upper-boundary, an investigation of the upper-boundary can be crucial.

For any arrival rate vector $\lambda$, we can define a norm-like function with respect to the capacity region, which we will loosely call schedule norm.

Definition 4. Given a link set $L$, the schedule norm of an arrival rate vector $\lambda$, denoted $\|\lambda\|_{s}$, is given by

$$
\|\lambda\|_{s}=\inf \left\{k \mid \exists \mu \in C o\left(M_{L}\right), k \mu \geq \lambda\right\} .
$$

Here, $\mu$ is interpreted as a service rate vector. If the links are endowed with queued data proportional to $\lambda$ and there are no 
further arrivals, then $\|\lambda\|_{s}$ is the shortest time to drain all data among all possible schedules. We next show that the schedule norm is equal to the weighted fractional chromatic number.

Lemma 7. For any arrival rate vector $\lambda,\|\lambda\|_{s}=\chi_{f}(G, \lambda)$.

Proof: The case of $\lambda=0$ is trivial. We only consider the case where $\lambda \neq 0$. Since $\operatorname{Co}\left(M_{L}\right)$ is a closed set, it can be shown easily the set in Definition $4,\left\{k \mid \exists \mu \in \operatorname{Co}\left(M_{L}\right), k \mu \geq\right.$ $\lambda\}$, is closed, and the infimum is achieved. Hence, for any arrival rate vector $\lambda$, there is a $\mu \in \operatorname{Co}\left(M_{L}\right)$ such that $\|\lambda\|_{s} \mu \geq \lambda$. Since $\mu \in \operatorname{Co}\left(M_{L}\right), \mu=M_{L} \alpha$ for some $\alpha \geq 0$ and $\sum_{i=1}^{\left|M_{L}\right|} \alpha_{i}=1$. Let $\beta_{i}=\|\lambda\|_{s} \alpha_{i}$ for each $i$; $\beta$ is feasible for the optimization problem to find $\chi_{f}(G, \lambda)$. Hence, $\|\lambda\|_{s} \geq \chi_{f}(G, \lambda)$. To show the other inequality, we let $\mu=M_{L} \frac{\beta^{\lambda}}{\chi_{f}(G, \lambda)}$, where $\beta^{\lambda}$ is an optimal solution to the problem of finding $\chi_{f}(G, \lambda)$. Then $\mu \in \operatorname{Co}\left(M_{L}\right)$ and $\chi_{f}(G, \lambda) \mu \geq \lambda$. Therefore, $\|\lambda\|_{s} \leq \chi_{f}(G, \lambda)$.

Corollary 2. The capacity region is the set containing all the vectors whose schedule norms are less than or equal to 1. The interior of the capacity region is the set containing all the vectors whose schedule norms are less than 1.

In [15], the length of a vector respect to a convex region $\Theta$ is defined as:

$$
\|x\|_{\Theta}=\frac{1}{\sup \{k \mid k \geq 0, k x \in \Theta\}} .
$$

Lemma 8. The definition of the schedule norm is identical to the definition in (3), if we let $\Theta=\Lambda$, i.e:

$$
\|x\|_{s}=\|x\|_{\Lambda} \text {. }
$$

Proof: Note that $\|x\|_{\Lambda}=1 / \psi_{x}^{*}$ by Definition 1 . By applying Lemma 3 and Lemma 7, we obtain the desired equality.

Set $\sigma$-local pooling is studied in [35]. It has many interesting properties and is related to $\sigma$-local pooling defined in [18]. We will show the relationship between the set $\sigma$-local pooling factor and the schedule norm. Let $L$ be any non-empty link set. For convenience, let

$$
\Theta_{L}=\left\{\sigma \mid \sigma \mu_{L} \ngtr \nu_{L}, \text { for all } \mu_{L}, \nu_{L} \in C o\left(M_{L}\right)\right\} .
$$

The compliment of $\Theta_{L}$ is

$$
\Theta_{L}^{c}=\left\{\sigma \mid \sigma \mu_{L}>\nu_{L}, \text { for some } \mu_{L}, \nu_{L} \in \operatorname{Co}\left(M_{L}\right)\right\} .
$$

Definition 5. Given a non-empty set of links $L$, we say $L$ has $a$ set $\sigma$-local pooling factor $\sigma_{L}$ if the following holds.

$$
\begin{aligned}
\sigma_{L} & :=\sup \left\{\sigma \mid \sigma \in \Theta_{L}\right\} \\
& :=\inf \left\{\sigma \mid \sigma \in \Theta_{L}^{c}\right\} .
\end{aligned}
$$

It has been shown that the set $\sigma$-local pooling factor is equal to the optimal solution of the following problem.

$$
\begin{gathered}
\min _{\sigma, \mu_{L}, \nu_{L}} \sigma \\
\text { subject to } \sigma \mu_{L} \geq \nu_{L} \\
\\
\mu_{L}, \nu_{L} \in \operatorname{Co}\left(M_{L}\right) .
\end{gathered}
$$

Let $K \subseteq L$. For any $|K|$-dimensional vector $\lambda$, we can define an extended $|L|$-dimensional vector $\lambda_{L}$ by setting all the extended components to be 0 . Conversely, for any $|L|$ dimensional vector $\mu$, we can create a $|K|$-dimensional vector by restriction, and we denote it by $[\mu]_{K}$.

Lemma 9. The set $\sigma$-local pooling factor satisfies: $\sigma_{K}=$ $\min \left\{\left\|\lambda_{L}\right\|_{s} \mid \lambda \in \operatorname{Co}\left(M_{K}\right)\right\}$.

Proof: For any $\lambda \in \operatorname{Co}\left(M_{K}\right), 0<\left\|\lambda_{L}\right\|_{s} \leq 1$. Letting $k=1 /\left\|\lambda_{L}\right\|_{s}$, we have $k \geq 1$ and $\left\|k \lambda_{L}\right\|_{s}=1$. Thus there is a vector $\mu \in \operatorname{Co}\left(M_{L}\right)$ such that $\mu \geq k \lambda_{L}$. Furthermore, there is a vector $\gamma \in \operatorname{Co}\left(M_{K}\right)$ satisfing $\gamma \geq[\mu]_{K}$. Then $\gamma \geq k \lambda$. Thus $1 / k \geq \sigma_{K}$. Therefore, $\sigma_{K} \leq \min \left\{\left.|| \lambda_{L}\right|_{s} \mid \lambda \in\right.$ $\left.\operatorname{Co}\left(M_{K}\right)\right\}$.

In the problem of set $\sigma$-local pooling, there is a $\mu, \nu \in$ $\operatorname{Co}\left(M_{K}\right)$ such that $\nu \leq \sigma_{K} \mu$. Let $\lambda=\nu$, we obtain that $\left\|\lambda_{L}\right\|_{s} \leq \sigma_{K}$. Therefore, $\min \left\{\left\|\lambda_{L}\right\|_{s} \mid \lambda \in \operatorname{Co}\left(M_{K}\right)\right\} \leq \sigma_{K}$.

The lemma shows that the set $\sigma$-local pooling factor is equal to the minimum schedule norm of all vectors in $\operatorname{Co}\left(M_{K}\right)$. The set $\sigma$-local pooling factor for set $K$ contains information about the shape of $\operatorname{Co}\left(M_{K}\right)$, as illustrated by the next corollary.

Corollary 3. When $\sigma_{L}=1$, any $\lambda \in \operatorname{Co}\left(M_{L}\right)$ satisfies $\|\lambda\|_{s}=1$.

Lemma 10. For a given set $L$ and any two vector $\lambda_{1}, \lambda_{2} \in$ $\operatorname{Co}\left(M_{L}\right)$, we have $\sigma_{L}\left(\left\|\lambda_{1}\right\|_{s}+\left\|\lambda_{2}\right\|_{s}\right) \leq\left\|\lambda_{1}+\lambda_{2}\right\|_{s}$.

Proof: Suppose there exist two vectors $\lambda_{1}, \lambda_{2} \in \operatorname{Co}\left(M_{L}\right)$ and $\sigma_{L}\left(\left\|\lambda_{1}\right\|_{s}+\left\|\lambda_{2}\right\|_{s}\right)>\left\|\lambda_{1}+\lambda_{2}\right\|_{s}$. Then, $2 \sigma_{L}>\| \lambda_{1}+$ $\lambda_{2} \|_{s}$ or $\sigma_{L}>\left\|1 / 2 \lambda_{1}+1 / 2 \lambda_{2}\right\|_{s}$. Since $1 / 2 \lambda_{1}+1 / 2 \lambda_{2} \in$ $\operatorname{Co}\left(M_{L}\right)$, this contradicts Lemma 9 .

Lemma 11. The upper-boundary satisfies $\mathscr{U} \subseteq \operatorname{Co}\left(M_{L}\right)$.

Proof: By the definition of $\mathscr{U}$, any $\lambda \in \mathscr{U}$ satisfies $\lambda \in$ $\Lambda$. Then, $\lambda \leq \mu$ for some $\mu \in C o\left(M_{L}\right)$. Thus, $\lambda=\mu$, which implies that $\lambda \in \operatorname{Co}\left(M_{L}\right)$ and the lemma holds.

Lemma 12. The upper-boundary satisfies $\mathscr{U}=C o\left(M_{L}\right)$ if and only if $\sigma_{L}=1$.

Proof: First, we will prove that if $\mathscr{U}=\operatorname{Co}\left(M_{L}\right)$ then $\sigma_{L}=1$. Suppose $\sigma_{L}<1$. There exist two vectors $\mu, \nu \in \operatorname{Co}\left(M_{L}\right)$ such that $\sigma_{L} \mu \geq \nu$. Then, $\nu \notin \mathscr{U}$, which is a contradiction.

Next, we will show that if $\sigma_{L}=1$, then $\mathscr{U}=\operatorname{Co}\left(M_{L}\right)$. From Lemma 11, we already know that $\mathscr{U} \subseteq C o\left(M_{L}\right)$. We only need to show $\operatorname{Co}\left(M_{L}\right) \subseteq \mathscr{U}$. Suppose $\operatorname{Co}\left(M_{L}\right) \nsubseteq \mathscr{U}$, then there exists a $\nu \in \operatorname{Co}\left(\bar{M}_{L}\right)$ and $\nu \notin \mathscr{U}$. Since $\nu \in \Lambda$, there must be a $\mu \in \operatorname{Co}\left(M_{L}\right)$ such that $\mu \geq \nu$ and $\mu \neq \nu$. Then, there is a partition of set $L$ into, say $P$ and $Q$, such that $P \cap Q=\emptyset, P \cup Q=L$ and $[\mu]_{Q}=[\nu]_{Q},[\mu]_{P}>[\nu]_{P}$. If $Q=\emptyset$, then there must be a $\sigma<1$ such that $\sigma \mu \geq \nu$. This contradicts $\sigma_{L}=1$. Suppose $Q \neq \emptyset$. If for all $i \in Q$, $\mu_{i}=\nu_{i}=0$, then again, there must be a $\sigma<1$ such that $\sigma \mu \geq \nu$.

We next consider the remaining case. Pick an $i \in Q$ such that $\mu_{i}=\nu_{i}>0$. For this $i$, there must be a schedule $s$ in 
which link $i$ is not activated. We choose a small enough $\epsilon$ and let $\nu^{\prime}=(1-\epsilon) \nu+\epsilon s$. Since $\nu, s \in C o\left(M_{L}\right)$, we have $\nu^{\prime} \in C o\left(M_{L}\right)$. Next, set $P \leftarrow P \cup\{i\}$ and $Q \leftarrow Q-\{i\}$. This operation can be repeated until either $Q=\emptyset$ or $[\mu]_{Q}=$ $\left[\nu^{\prime}\right]_{Q}=0$. Then, there must be a $\sigma<1$ such that $\sigma \mu \geq \nu^{\prime}$, which contradicts $\sigma_{L}=1$.

This lemma implies that whenever $\sigma_{L}=1$, the upperboundary is convex and equal to $C o\left(M_{L}\right)$. Then, any ray from the origin can only intersect $\operatorname{Co}\left(M_{L}\right)$ at one point.

\section{CONCLUSION AND FUture WORK}

In this paper, we investigated the capacity region of a wireless network in a meticulous manner. In particular, we defined the capacity region using the weighted fractional coloring problem and used the derived relations to address the complexity of scheduling deterministic arrivals. The choice of deterministic arrivals was due to its immediate connection to the randomized counterpart and the ease of applying the complexity theory. Under the deterministic setting, we showed that the MWIS-based algorithm stabilizes the queues with efficient bounds on the queue sizes. We also investigated interesting sections of the capacity region and defined the upper-boundary. We related the $\sigma$-local pooling factor to the weighted fractional coloring problem. We characterized the upper-boundary under the set local pooling condition.

There are many remaining questions. First, the connection between the MWIS problem and fractional coloring in the scheduling context has not been established in a rigorous manner. Second, the applicability of the results to the state of art control algorithms requires connecting deterministic arrivals with probabilistic ones. Third, we believe that set local pooling (and local pooling in general) is related to the complexity of the MWIS problem in graphs. Our immediate concern will be addressing these issues.

\section{REFERENCES}

[1] P. Gupta and P. R. Kumar, "The capacity of wireless networks," IEEE Transactions on Information Theory, vol. 46, no. 2, pp. 388-404, 2000.

[2] S. Borbash and A. Ephremides, "Wireless link scheduling with power control and SINR constraints," IEEE Transactions on Information Theory, vol. 52, no. 11, pp. 5106-5111, Nov. 2006.

[3] G. Mergen and L. Tong, "Stability and capacity of regular wireless networks," IEEE Transactions on Information Theory, vol. 51, no. 6, pp. 1938-1953, June 2005.

[4] L. Tassiulas and A. Ephremides, "Stability properties of constrained queueing systems and scheduling policies for maximum throughput in multihop radio networks," IEEE Transactions on Automatic Control, vol. 37, no. 12, pp. 1936-1948, Dec 1992.

[5] L. Tassiulas, "Linear complexity algorithms for maximum throughput in radio networks and input queued switches," in Proceedings of IEEE INFOCOM, 1998.

[6] Y. Yi, A. Proutière, and M. Chiang, "Complexity in wireless scheduling: Impact and tradeoffs," in MobiHoc, 2008.

[7] S. Sanghavi, L. Bui, and R. Srikant, "Distributed link scheduling with constant overhead," SIGMETRICS Perform. Eval. Rev., vol. 35, no. 1, pp. 313-324, 2007.

[8] A. Eryilmaz, O. Asuman, and E. Modiano, "Polynomial complexity algorithms for full utilization of multi-hop wireless networks," in Proceedings of INFOCOM 2007, May 2007, pp. 499-507.

[9] E. Modiano, D. Shah, and G. Zussman, "Maximizing throughput in wireless networks via gossiping," SIGMETRICS Perform. Eval. Rev., vol. 34, no. 1, pp. 27-38, 2006.
[10] A. Brzezinski, G. Zussman, and E. Modiano, "Enabling distributed throughput maximization in wireless mesh networks: a partitioning approach," in Proceedings of ACM Mobicom, 2006, pp. 26-37.

[11] L. Jiang and J. Walrand, "A CSMA distributed algorithm for throughput and utility maximization in wireless networks," University of California, Berkeley, Tech. Rep., 2008.

[12] J. Liu, Y. Yi, A. Proutière, M. Chiang, and H. V. Poor, "Maximizing utility via random access without message passing," Princeton University, Tech. Rep., 2008.

[13] S. Rajagopalan and D. Shah, "Distributed algorithm and reversible network," in Proccedings of CISS, March 2008, pp. 498-502.

[14] X. Lin, N. B. Shroff, and R. Srikant, "The impact of imperfect scheduling on cross-layer rate control in wireless networks," IEEE/ACM Transaction on Networking, vol. 14, no. 2, pp. 302-315, April 2006.

[15] X. Lin and S. B. Rasool, "Constant-time distributed scheduling policies for ad hoc wireless networks," in Proceedings of the IEEE CDC, 2006.

[16] C. Joo and N. Shroff, "Performance of random access scheduling schemes in multi-hop wireless networks," in Proceedings of IEEE INFOCOM, 2007.

[17] X. Wu, R. Srikant, and J. R. Perkings, "Scheduling efficiency of distributed greedy scheduling algorithms in wireless networks," in Proceedings of IEEE INFOCOM, 2006.

[18] C. Joo, X. Lin, and N. B. Shroff, "Understanding the capacity region of the greedy maximal scheduling algorithm in multi-hop wireless networks," in Proceedings of IEEE INFOCOM, 2008.

[19] C. Joo, "A local greedy scheduling scheme with provable performance guarantee," in Proceedings of MobiHoc, 2008, pp. 111-120.

[20] A. Dimakis and J. Walrand, "Sufficient conditions for stability of longest-queue-first scheduling: Second-order properties using fluid limits," Advances in Applied Probability, vol. 38, pp. 505-521, 2006.

[21] G. Zussman, A. Brzezinski, and E. Modiano, "Multihop local pooling for distributed throughput maximization in wireless networks," in Proccedings of IEEE INFOCOM, April 2008, pp. 1139-1147.

[22] A. Brzezinski, G. Zussman, and E. Modiano, "Local pooling conditions for joint routing and scheduling," in Information Theory and Applications Workshop, 2008, pp. 499-506.

[23] M. Neely, "Delay analysis for maximal scheduling in wireless networks with bursty traffic," in Proccedings of IEEE INFOCOM, April 2008, pp. 6-10.

[24] M. J. Neely, E. Modiano, and C. E. Rohrs, "Tradeoffs in delay guarantees and computation complexity for nxn packet switches," in Proccedings of CISS, 2002.

[25] D. Shah and M. Kopikare, "Delay bounds for approximate maximum weight matching algorithms for input queued switches," in Proccedings of IEEE INFOCOM, 2002, pp. 1024-1031.

[26] B. Hajek and G. Sasaki, "Link scheduling in polynomial time," IEEE Transactions on Information Theory, vol. 34, no. 5, pp. 910-917, Sep 1988.

[27] S. Khanna and K. Kumaran, "On wireless spectrum estimation and generalized graph coloring," in Proceedings of IEEE INFOCOM, vol. 3 , Mar-2 Apr 1998, pp. 1273-1283 vol.3.

[28] B. Awerbuch and F. T. Leighton, "A simple local-control approximation algorithm for multicommodity flow," in IEEE Symposium on Foundations of Computer Science, 1993, pp. 459-468.

[29] U. Feige and J. Kilian, "Zero knowledge and the chromatic number," in Proceedings of CCC. Washington, DC, USA: IEEE Computer Society, 1996, p. 278.

[30] M. Grötschel, L. Lovász, and A. Schrijver, "The ellipsoid method and its consequences in combinatorial optimization," Combinatorica, vol. 1, pp. 169-197, 1981.

[31] M. Grötschel, L. Lovász, and A. Schrijver, Geometric Algorithms and Combinatorial Optimization, second corrected edition ed., ser. Algorithms and Combinatorics. Springer, 1993, vol. 2.

[32] K. Jansen, "Approximate strong separation with application in fractional graph coloring and preemptive scheduling," Theor. Comput. Sci., vol. 302, no. 1-3, pp. 239-256, 2003.

[33] K. Jansen and L. Porkolab, "On preemptive resource constrained scheduling: Polynomial-time approximation schemes," SIAM J. Discret. Math., vol. 20, no. 3, pp. 545-563, 2006.

[34] C. Lund and M. Yannakakis, "On the hardness of approximating minimization problems," J. ACM, vol. 41, no. 5, pp. 960-981, 1994.

[35] B. Li, C. Boyaci, and Y. Xia, "A refined performance characterization of longest-queue-first policy in wireless networks," in Proceedings of MobiHoc. New York, NY, USA: ACM, 2009, pp. 65-74. 